Stephan Meckel

Ralf Mekle

Christian Taschner

Sven Haller

Klaus Scheffler

Ernst-Wilhelm Radue

Stephan G. Wetzel

\section{Time-resolved 3D contrast-enhanced MRA with GRAPPA on a 1.5-T system for imaging of craniocervical vascular disease: initial experience}

Received: 28 June 2005

Accepted: 2 November 2005

Published online: 11 March 2006

C Springer-Verlag 2006
S. Meckel $(\bowtie) \cdot$ C. Taschner

S. Haller - E.-W. Radue - S. G. Wetzel

Division of Neuroradiology/

Department of Radiology,

University Hospital Basel,

Petersgraben 4,

Basel 4031, Switzerland

e-mail: meckels@uhbs.ch

R. Mekle $\cdot$ K. Scheffler

MR Physics,

Department of Medical Radiology,

University Hospital Basel,

Petersgraben 4,

Basel 4031, Switzerland

\begin{abstract}
Introduction: For three-dimensional (3D) imaging with magnetic resonance angiography (MRA) of the cerebral and cervical circulation, both a high temporal and a high spatial resolution with isovolumetric datasets are of interest. In an initial evaluation, we analyzed the potential of contrast-enhanced (CE) time-resolved 3D-MRA as an adjunct for neurovascular MR imaging. Methods: In ten patients with various cerebrovascular disorders and vascularized tumors in the cervical circulation, high-speed MR acquisition using parallel imaging with the GeneRalized Autocalibrating Partially Parallel Acquisitions (GRAPPA) algorithm on a 1.5-T system with a temporal resolution of $1.5 \mathrm{~s}$ per dataset and a nearly isovolumetric spatial resolution was applied. The results were assessed and compared with those from conventional MRA and digital subtraction angiography (DSA). Results: CE timeresolved 3D-MRA enabled the visualization and characterization of
\end{abstract}

high-flow arteriovenous shunts in cases of vascular malformations or hypervascularized tumors. In stenoocclusive disease, the method provided valuable additional information about altered vessel perfusion compared to standard MRA techniques such as time-of-flight (TOF) MRA. The use of a nearly isovolumetric voxel size allowed a free-form interrogation of $3 \mathrm{D}$ datasets. Its comparatively low spatial resolution was found to be the major limitation. Conclusion: In this preliminary analysis, CE timeresolved 3D-MRA was revealed to be a promising complementary MRA sequence that enabled the visualization of contrast flow dynamics in various types of neurovascular disorders and vascularized cervical tumors.

Keywords Time-resolved contrastenhanced MR angiography .

Parallel imaging · 3D MRA imaging ·

Cerebral arteriovenous malformations $\cdot$ Cerebrovascular disease

\section{Introduction}

The ideal tool for imaging of the cerebral vasculature should possess the following features: high spatial and high temporal resolution, 3D volumetric information, a high signal-to-noise ratio (SNR), a broad and inexpensive availability, fast performance and an acceptable degree of safety. Digital subtraction angiography (DSA), still the standard of reference for cerebrovascular imaging, comes close to these objectives. However, as DSA is an invasive procedure, there is an accompanying low risk $(<1 \%)$ of potentially disabling neurological complications [1]. Other disadvantages are high cost and $\mathrm{x}$-ray dose exposure for the patient and the radiologist/staff.

Standard magnetic resonance angiography (MRA) using time-of-flight (TOF) techniques provides acceptable spatial resolution that is sufficient to diagnose a variety of disorders; however, it does not provide information on contrast flow dynamics that is important for the diagnosis and characterization of numerous cerebrovascular disor- 
ders, especially those with arteriovenous (AV) shunting, e.g., dural AV fistula (DAVF).

Two-dimensional contrast-enhanced time-resolved MR projection angiography (2D-MRPA) using complex subtraction yields images showing vascular enhancement at a high temporal resolution $[2,3]$. This technique has proven to be highly effective for demonstrating neurovascular diseases, in particular DAVFs $[4,5]$. However, the lack of volumetric information, the necessity for repeated injections of contrast medium in separately acquired planes (e.g., coronal, sagittal), signal cancellations along the slab, as well as superposition of different supraaortic vessels are important limitations.

Recently, parallel imaging techniques for MR data acquisition using multielement receiver coil arrays have been introduced. These are methods that partially shift the burden of spatial encoding from magnetic field gradients to parallel receive coils allowing faster image acquisition.

We implemented a 3D MRA technique using the GeneRalized Autocalibrating Partially Parallel Acquisitions (GRAPPA) algorithm for parallel imaging in order to achieve a sufficiently high temporal resolution [6]. We termed this method time-resolved 3D-MRA.

We aimed to evaluate this technique in comparison to DSA and standard MRA techniques, namely TOF-MRA and contrast-enhanced MRA (CE-MRA), to assess vascular diseases in the cervical and cerebral circulation, including vascular malformations, highly vascularized tumors and steno-occlusive disease. We present an initial evaluation of the comparative diagnostic role of isotropic

Table 1 Comparative analysis of time-resolved 3D-MRA findings with DSA and other MRA techniques ( $B A$ basilar artery, $C S$ cavernous sinus, $D V A$ developmental venous angioma, $E C A$ external

posterior cerebral artery, PICA posterior inferior cerebral artery, SS sigmoid sinus, $S S S$ superior sagittal sinus, $T S$ transverse sinus, $V A$ carotid artery, $I C A$ internal carotid artery, $O A$ occipital artery, $P C A$ vertebral artery)

\begin{tabular}{|c|c|c|c|c|c|c|}
\hline $\begin{array}{l}\text { Patient } \\
\text { no. }\end{array}$ & $\begin{array}{l}\text { Age } \\
\text { (years)/ } \\
\text { sex }\end{array}$ & Category & Diagnosis & Major DSA findings & $\begin{array}{l}\text { Time-resolved 3D- } \\
\text { MRA findings } \\
\text { additional to MR/ } \\
\text { MRA }^{\text {a }}\end{array}$ & $\begin{array}{l}\text { Time-resolved 3D-MRA } \\
\text { findings missing } \\
\text { compared to DSA/ } \\
\text { MRA }^{\text {a }}\end{array}$ \\
\hline 1 & $56 / \mathrm{F}$ & $\begin{array}{l}\text { Vascular } \\
\text { malforma- } \\
\text { tion }\end{array}$ & Complex DAVF (type 4) & $\begin{array}{l}\text { Various ECA feeders. Drainage } \\
\text { into ectatic cortical veins with } \\
\text { reflux into SSS }\end{array}$ & $\begin{array}{l}\text { Arterial feeders. Cor- } \\
\text { tical drainage with } \\
\text { reflux into SSS }\end{array}$ & $\begin{array}{l}\text { Detailed feeders and } \\
\text { drainage (super- } \\
\text { selective DSA) }\end{array}$ \\
\hline 2 & $60 / \mathrm{M}$ & & DAVF TS (type 1) & $\begin{array}{l}\text { Major OA feeders. Small VA } \\
\text { feeders. Antegrade drainage in } \\
\text { TS. No cortical reflux }\end{array}$ & $\begin{array}{l}\text { Presence of DAVF. } \\
\text { Arterial feeders, an- } \\
\text { tegrade venous } \\
\text { drainage }\end{array}$ & $\begin{array}{l}\text { Small VA feeders } \\
\text { (selective DSA) }\end{array}$ \\
\hline 3 & $50 / \mathrm{M}$ & & $\begin{array}{l}\text { Recurrent DAVF TS/SS } \\
\text { (type 2a). Post-transve- } \\
\text { nous embolization }\end{array}$ & $\begin{array}{l}\text { Distal TS occluded. Recurrent } \\
\text { DAVF at proximal TS. No } \\
\text { cortical reflux }\end{array}$ & $\begin{array}{l}\text { Recurrent DAVF. Ar- } \\
\text { terial feeders, venous } \\
\text { drainage pattern }\end{array}$ & $\begin{array}{l}\text { Detailed arterial feeders } \\
\text { (superselective DSA) }\end{array}$ \\
\hline 4 & $63 / \mathrm{M}$ & & $\begin{array}{l}\text { CS fistula (Barrow C). } \\
\text { Post-embolization }\end{array}$ & Total fistula occlusion & Fistula occlusion & None \\
\hline 5 & $52 / \mathrm{M}$ & & DVA & $\begin{array}{l}\text { Large transmedullary vein. No } \\
\text { AV shunts. Tiny caput medusae }\end{array}$ & No AV shunts & $\begin{array}{l}\text { Tiny caput medusae } \\
\text { (DSA) }\end{array}$ \\
\hline 6 & $45 / \mathrm{M}$ & $\begin{array}{l}\text { Steno-occlu- } \\
\text { sive disease }\end{array}$ & $\begin{array}{l}\text { Suspected venous sinus } \\
\text { thrombosis }\end{array}$ & N/A & Flow dynamics in TS & None \\
\hline 7 & $43 / \mathrm{M}$ & & $\begin{array}{l}\text { Follow-up after coil oc- } \\
\text { clusion of dissecting } \\
\text { VA aneurysm in V3/V4 } \\
\text { segment }\end{array}$ & $\begin{array}{l}\text { Persistent VA occlusion. Cross- } \\
\text { over perfusion of posterior in- } \\
\text { ferior cerebellar artery (PICA) }\end{array}$ & Dynamic flow pattern & $\begin{array}{l}\text { Right PICA not } \\
\text { visualized (MRA) }\end{array}$ \\
\hline 8 & $76 / \mathrm{M}$ & & $\begin{array}{l}\text { Persistent primitive tri- } \\
\text { geminal artery. Verte- } \\
\text { brobasilar ischemia }\end{array}$ & $\begin{array}{l}\text { Retrograde PCA/BA perfusion. } \\
\text { VAs fade away (aortic injection) }\end{array}$ & $\begin{array}{l}\text { Retrograde perfusion } \\
\text { of PCAs/BA. VAs } \\
\text { not connected to BA }\end{array}$ & None \\
\hline 9 & $62 / F$ & Tumor & Carotid paraganglioma & $\begin{array}{l}\text { Large tumor blush inside carotid } \\
\text { bifurcation. ICA feeders only }\end{array}$ & Tumor filling pattern & $\begin{array}{l}\text { Detailed supply } \\
\text { (superselective DSA) }\end{array}$ \\
\hline 10 & $78 / \mathrm{F}$ & & $\begin{array}{l}\text { Jugular paraganglioma. } \\
\text { Partial embolization }\end{array}$ & $\begin{array}{l}\text { Arterial tumor blush. Different } \\
\text { ECA feeders. Small VA, ICA, } \\
\text { and PICA feeders }\end{array}$ & $\begin{array}{l}\text { Tumor filling pattern, } \\
\text { retrograde venous } \\
\text { drainage }\end{array}$ & $\begin{array}{l}\text { Detailed feeders } \\
\text { (superselective DSA) }\end{array}$ \\
\hline
\end{tabular}

${ }^{a}$ Conventional MR/MRA sequences, e.g., TOF or whole-brain CE-MR venography 
time-resolved 3D-MRA as an adjunct to conventional MR and MRA techniques.

\section{Methods and subjects}

Patients

Ten consecutive patients (four females and six males) aged 43-78 years (mean 59.8 years) were examined using timeresolved 3D-MRA as part of the standard MR examination protocol. The decision to perform an additional timeresolved 3D-MRA was made by an experienced neuroradiologist prior to the scan on the basis of the clinical findings and suspected disorder. DSA was performed in nine patients with various neurovascular diseases as listed in Table 1. Nine patients underwent 3D-MRA using a TOF technique. In addition, for selected patients, a CE-MRA, a 2D-TOF venography or both were acquired.

\section{MR protocol}

\section{Time-resolved 3D-MRA}

All MRI examinations were performed on a 1.5-T Avanto Scanner (Siemens Medical Solutions, Erlangen, Germany) equipped with a SQ gradient system (slew rate $200 \mathrm{~T} / \mathrm{s} / \mathrm{m}$; gradient strength $40 \mathrm{mT} / \mathrm{m}$ ). A 12-channel head coil was used for all MRI data acquisitions.

The time-resolved 3D-MRA technique used during this study is based on a 3D radiofrequency (rf)-spoiled fast low angle shot (FLASH) sequence. A slab-selective rectangular rf pulse was applied for excitation and gradient timing was optimized to yield the following scan parameters: TR/TE 1.74/0.64, flip angle $\alpha 15^{\circ}$, bandwidth (BW) $1220 \mathrm{~Hz} /$ pixel, field-of-view (FOV) $255 \times 255 \mathrm{~mm}$, matrix $128 \times 128$, slices/slab 56, slab thickness $123.2 \mathrm{~mm}$, number of averages (NA) 1, phase resolution $100 \%$, slice resolution $64 \%$, phase partial Fourier factor $6 / 8$, and slice partial Fourier factor 6/8. Parallel imaging was utilized using the GRAPPA algorithm along two dimensions. Beside spatial resolution (voxel volume) and signal acquisition time, SNR in parallel imaging also depends on the spatial arrangement of the coil array (geometry factor g), the acceleration factor $\mathrm{R}$ and the reconstruction algorithm [7]. Accordingly, increasing the acceleration factor results in a decrease in SNR, which parallels the increase in temporal resolution. With the given geometry of our 12-channel head coil, the SNR and reconstruction artifacts became critical beyond an acceleration factor of 6 . An acceleration factor of 6 (acceleration factor of 3 and 30 reference lines along the phase encoding direction; acceleration factor of 2 and 16 reference lines along the partition encoding direction) was therefore selected as a trade-off between image quality and temporal resolution. The acquisition mode was sagittal with $29 \%$ slice over-sampling. Covering the entire head with these acquisitions, an in-plane resolution of $2.0 \times 2.0 \mathrm{~mm}$ with a slice thickness of $2.2 \mathrm{~mm}$ and a temporal resolution of $1.5 \mathrm{~s}$ per $3 \mathrm{D}$ dataset were obtained.

For selected cases $(n=3)$ where the exact position of the pathology was known from previous imaging and clinical data, targeted slab scanning was employed to focus on a region of interest. Maintaining the temporal resolution of $1.5 \mathrm{~s}$ per dataset, the number of slices per slab (32), the slab thickness $(50 \mathrm{~mm})$ and the FOV $(210 \times 210 \mathrm{~mm})$ were changed to obtain an improved isotropic voxel size of $1.6 \times 1.6 \times 1.6 \mathrm{~mm}$. Slice oversampling was increased to $88 \%$ to avoid infolding artifacts.

A single bolus of contrast agent $(0.5 \mathrm{M}$ gadoliniumDOTA) was administered using a power injector $(20 \mathrm{ml}$, $3 \mathrm{ml} / \mathrm{s}$ ). In total, a series of $253 \mathrm{D}$ datasets was acquired, where the acquisition of the first dataset was started simultaneously with the injection of the contrast agent bolus (TA $37.5 \mathrm{~s}$ ). The first acquisition was discarded and the second dataset was used as a mask. Subsequently, magnitude subtraction was used to remove any background signal from the $3 \mathrm{D}$ volumes. Automated maximum-intensity projected (MIP) reconstructions were generated in standard planes (coronal, sagittal).

\section{Spin-echo MR imaging, TOF-MRA/MR venography} and CE-MRA

All patients underwent standard MRI examination of the brain consisting of T1-weighted spin-echo (T1-SE) scans before and after Gd-DTPA administration, a T2-weighted turbo spin-echo (T2-TSE) scan and diffusion-weighted imaging (DWI).

In addition, 3D TOF-MRA was performed with the following parameters: TR/TE 39/5.21, ramped flip angle $\alpha$ $10-30^{\circ}$, nominal flip angle $\alpha 20^{\circ}$, FOV $200 \times 167 \mathrm{~mm}$, voxel size $0.6 \times 0.5 \times 1 \mathrm{~mm}$ and venous presaturation. Applying the GRAPPA algorithm (acceleration factor of 2,24 reference lines) resulted in a scan time of $4 \mathrm{~min} 38 \mathrm{~s}$. In five patients, 2D-TOF-MR venograms of coronal orientation were measured with the following scan parameters: TR/TE 26/7.2, flip angle $\alpha 60^{\circ}$, FOV $200 \times 188 \mathrm{~mm}$, in-plane resolution $0.8 \times 0.8 \mathrm{~mm}$, and slice thickness $4 \mathrm{~mm}$. Here, 45 slices were acquired in $3 \mathrm{~min} 5 \mathrm{~s}$.

For three patients, an additional high-resolution, wholebrain CE-MRA was obtained using a 3D rf-spoiled gradient echo (GRE) sequence (TR/TE 9/3.38, flip angle $\alpha 10^{\circ}$, voxel size $1.1 \times 0.9 \times 1.5 \mathrm{~mm}$ ) as previously described [8].

\section{Intraarterial DSA}

Diagnostic biplanar intraarterial DSA (Axiom Artis, Siemens Medical Solutions, Erlangen, Germany) was performed after catheterization of the common, external and 
internal carotid arteries (CCAs, ECAs, ICAs) and of the vertebral arteries (VAs) with a $4 \mathrm{~F}$ catheter via a femoral approach. In four patients, superselective catheterization of the external branches of the carotid artery was performed depending on the clinical setting.

\section{Evaluation}

Two experienced neuroradiologists in consensus evaluated images from all patients. Image analysis was performed in three steps. First, the conventional MR images including spin-echo T1 and T2 images, 2D- or 3D-TOF (or both) and, where available, CE-MRA, were analyzed and the main findings recorded. At this point, both observers were blinded to the findings of time-resolved 3D-MRA and DSA. All MR images were assessed on a 3D postprocessing workstation (Leonardo, Siemens Medical Solutions, Erlangen, Germany).

Second, images obtained from time-resolved 3D-MRA scans were assessed. Automatically in-line subtracted coronal and sagittal MIP reconstructions were used. In addition, observers had the opportunity to generate targeted MIP reconstructions along any desired obliquity. Any deviations from the conventional MR/MRA imaging findings (e.g., any additional or missing information) were recorded.

Fig. 1 a,b Sagittal MIP reconstructions from a 3D-MRA time-series showing early abnormal filling of ectatic cortical veins in the occipital region via three different feeders from external carotid artery branches (a). Retrograde flow into the superior sagittal sinus is noted on the image obtained during early venous phase (b). c The corresponding DSA image obtained from a common carotid artery injection during early arterial phase. d Superselective DSA with injection into the right external carotid artery. Two different middle meningeal artery branches can be identified as arterial feeders to the type 4 DAVF with direct shunting into dilated cortical veins. Venous drainage follows these ectatic veins into the superior sagittal sinus (black arrow). The third arterial feeder, a right occipital artery branch, is not visualized due to distal positioning of the catheter (compared with c)
Third, DSA images were assessed as the standard of reference. If DSA was not performed ( $n=1), \mathrm{CE}-\mathrm{MRA}$ and/ or TOF-MRA served as the standard of reference. The investigators then recorded findings missing on the timeresolved 3D-MRA images in comparison to DSA images. DSA images were evaluated on hard copies created from standard and superselective series of each examination.

The criteria used for the evaluation of conventional MRA, time-resolved 3D-MRA and DSA images were dependent on the clinical findings and questioned abnormalities. If a steno-occlusive disorder was considered, the degree of venous or arterial patency, the direction of flow and the presence and pattern of collateral flow were chosen as criteria. In contrast, if disorders with AV shunting, e.g., DAVFs, were suspected, the presence and pattern of feeding arteries and draining veins, as well as alternation of venous outflow and the presence of reflux into cortical veins, were used as criteria for image evaluation. DAVFs were graded according to the classification revised by Cognard et al. [9].

\section{Results}

All ten patients were categorized into three major groups of disease. All findings are summarized in Table 1 including
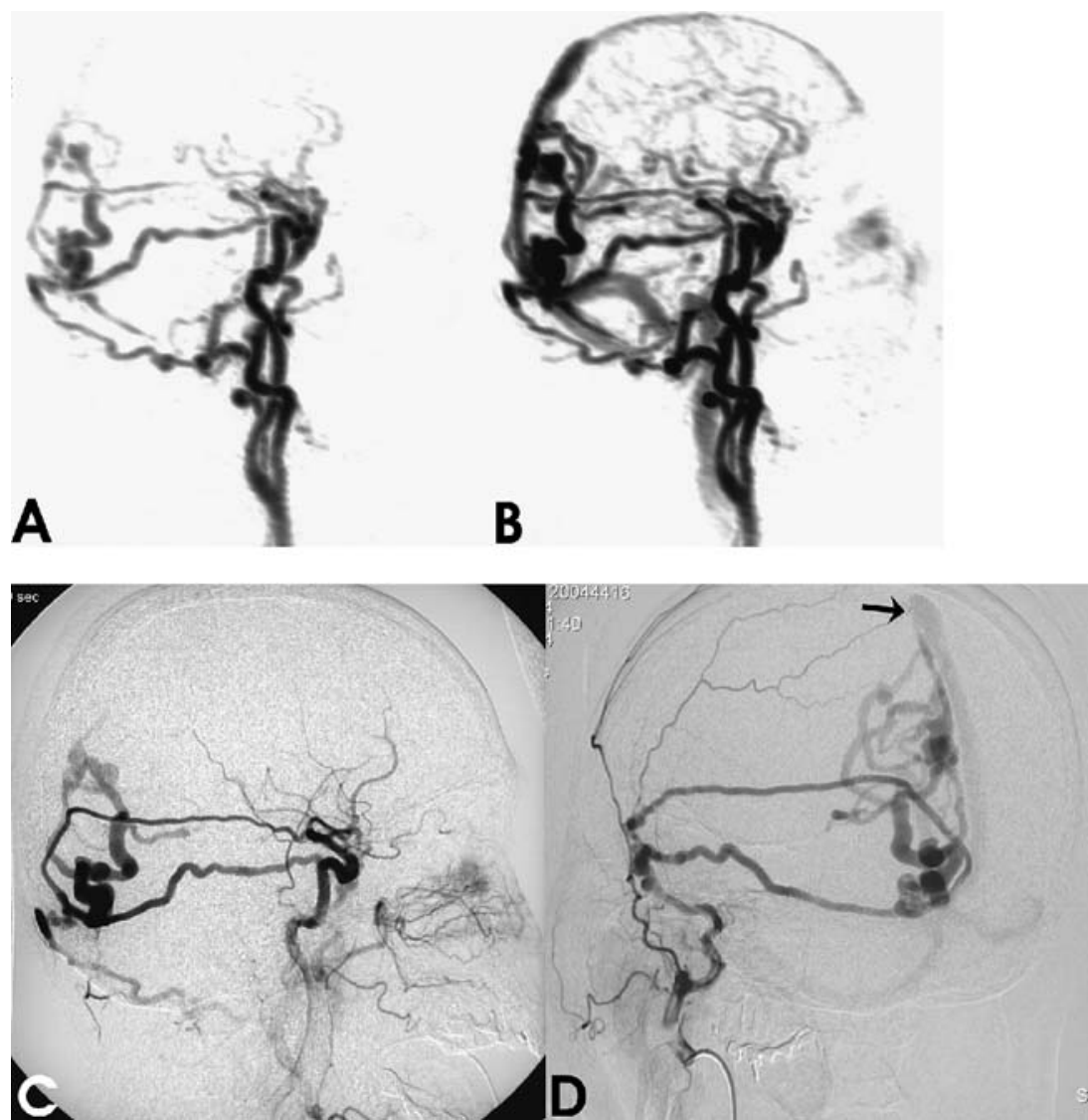
the results from the comparative assessment of different angiographic techniques.

\section{Vascular malformations}

Patient 1 presented clinically with visual aura and bilateral pulsatile tinnitus (Fig. 1). Standard MRI examination revealed large flow voids in the parasagittal occipital region that were suggestive of ectatic veins draining into the superior sagittal sinus; 3D-TOF MRA showed a prominent ipsilateral meningeal vessel. A complex DAVF was diagnosed on the basis of time-resolved 3D-MRA images showing three main feeders from the middle meningeal artery branches and the occipital artery with venous hypertension caused by cortical drainage via ectatic veins into the superior sagittal sinus (Cognard type 4). DSA verified the diagnosis; however, superselective catheterization of ECA branches allowed a more precise description of the arterial feeders and details of the cortical drainage pattern.

In a second patient (patient 2), 3D-TOF MRA showed areas of high signal intensity within the right transverse sinus that were of equivocal diagnostic relevance. Time-
Fig. 2 a,b Time-resolved 3DMRA images obtained during early arterial phase. On parasagittal thin MIP-reconstructed image the arterial feeding branches from right occipital artery with early drainage into the transverse sinus are depicted (a). A coronal targeted MIP reconstruction (b) focused on the transverse sinuses demonstrates early filling from the right occipital artery with occlusion of the distal part of right transverse sinus and crossover flow to the left side. c,d Intraarterial DSA images confirm the findings from time-resolved 3D-MRA. The lateral angiogram (c) shows main arterial feeders from the occipital artery with early drainage into the transverse sinus similar to that in a. On the selective angiogram with injection into the right occipital artery (d), AV shunting into the proximal right transverse sinus with cross flow to the left transverse sinus corresponding to MRA image b is best visualized. The distal part of the transverse sinus is coil-occluded. e Angiogram with injection into the contralateral left common carotid artery shows additional tiny arterial feeders to the fistula point that originate from the left occipital artery (black arrowheads)

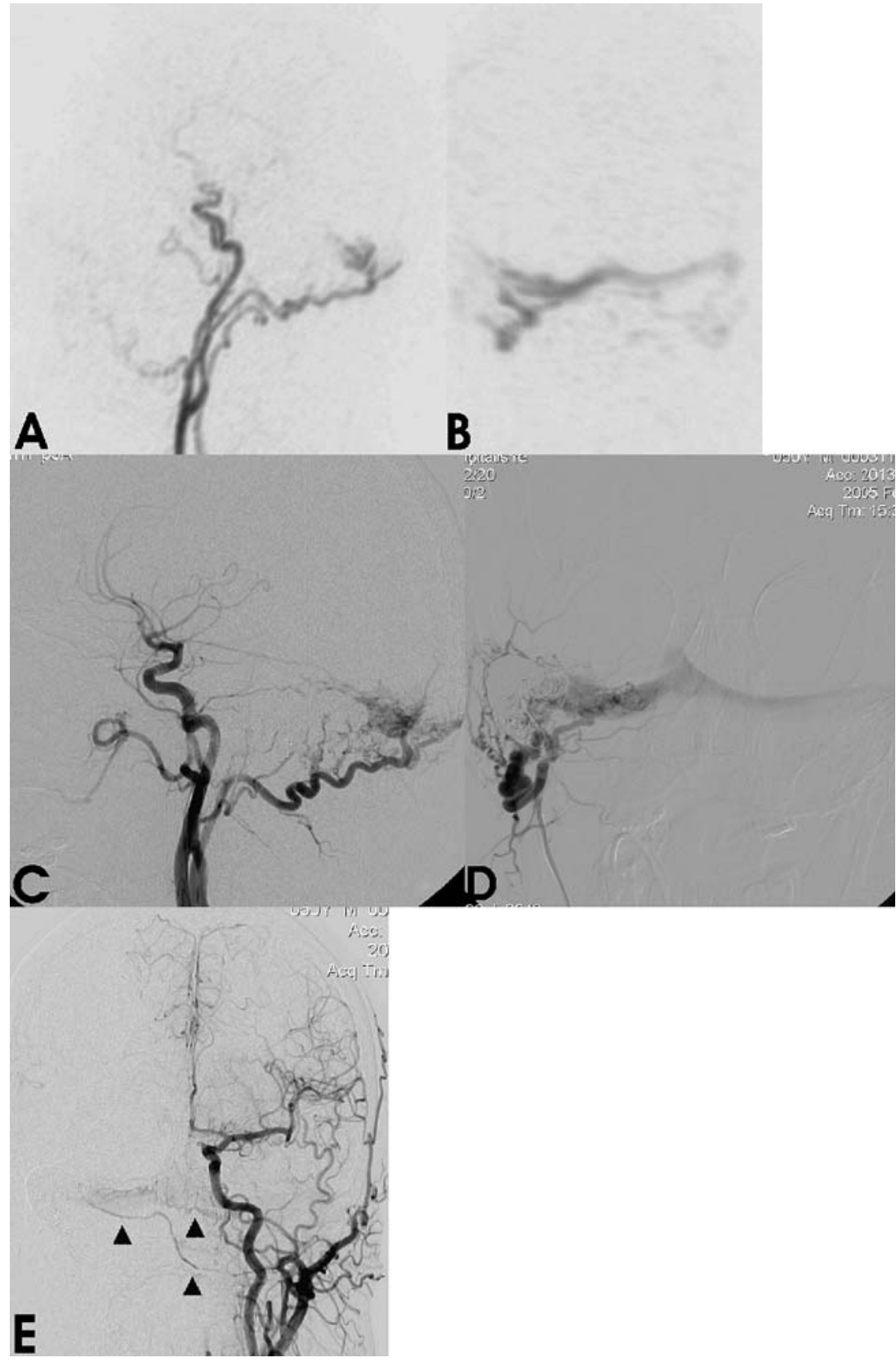


resolved 3D-MRA showed a DAVF at the right transverse sinus with antegrade venous flow (Cognard type 1) and arterial feeders from the right occipital artery. On DSA with selective injection into the right VA, small muscular branches originating from the V3 segment were identified as additional arterial feeders.

Two patients who had previously been treated for a DAVF by endovascular embolization were examined in the course of their post-interventional follow-up. Patient 3 had a DAVF (Cognard type 2a) at the junction of the right transverse/sigmoid sinus that had been occluded by transvenous coil embolization 1 year prior to the examination. Time-resolved 3D-MRA images showed occlusion of the right distal transverse sinus (Fig. 2). In addition, early filling of its proximal part via feeders from the right occipital artery with subsequent venous cross flow to the contralateral side was observed indicating a recurrence of the DAVF. The fistula point was proximal to the entry site of the vein of Labbé that showed antegrade filling. On DSA images with superselective catheterization of ECA branches, two small feeding arteries originating from the right middle meningeal artery and the contralateral occipital artery were detected in addition to the findings obtained from time-resolved 3D-MRA.

Patient 4, with a cavernous sinus fistula (Barrow type C), showed a complete exclusion of the fistula on 3D-MRA images 10 months after transarterial embolization which was verified by DSA.

In patient 5 , who presented with an acute onset of headache and diplopia, standard MRI revealed a large transmedullary vein extending from the subependymal white matter upwards into the superior sagittal sinus. Additional MR imaging was requested in order to exclude a mixed malformation that might be associated with an increased risk of hemorrhage. Both time-resolved 3DMRA and confirmatory DSA provided sufficient information to rule out a high-flow AV shunt in this venous angioma. Compared to DSA and contrast-enhanced T1weighted spin-echo images, a tiny caput medusae sign was not clearly visualized on images obtained from timeresolved 3D-MRA.

\section{Steno-occlusive diseases}

Three patients with different types of arterial and venous steno-occlusive disease were included in this group. Patient 6 , who presented with long-standing symptoms suggestive of venous sinus thrombosis, was examined with standard MRI, TOF MR venography, whole-brain CE-MRA and time-resolved 3D-MRA (Fig. 3). The 2D-TOF venogram did not reveal flow within the proximal part of the right transverse sinus (due either to occlusion or flow-related artifacts), whereas contrast enhancement within this part of the sinus was shown on the CE-MRA images. Venous blood flow was definitely indicated within the whole right transverse sinus on images obtained from an early venous dataset of the time-resolved 3D MR angiogram.

Arterial flow patterns in the posterior circulation were analyzed in two cases. In contrast to conventional MRA techniques, time-resolved 3D-MRA allowed the detection of flow direction in arterial segments adjacent to the site of stenosis or occlusion. Patient 7 underwent follow-up MRA imaging after endovascular coil occlusion for a dissecting aneurysm in the V3/V4 segment of the right VA (Fig. 4). 3D-TOF MRA showed a loss of signal at the site of the coil-occlusion and a tiny perfused lumen of the right PICA. Although a direct visualization of the tiny right PICA was
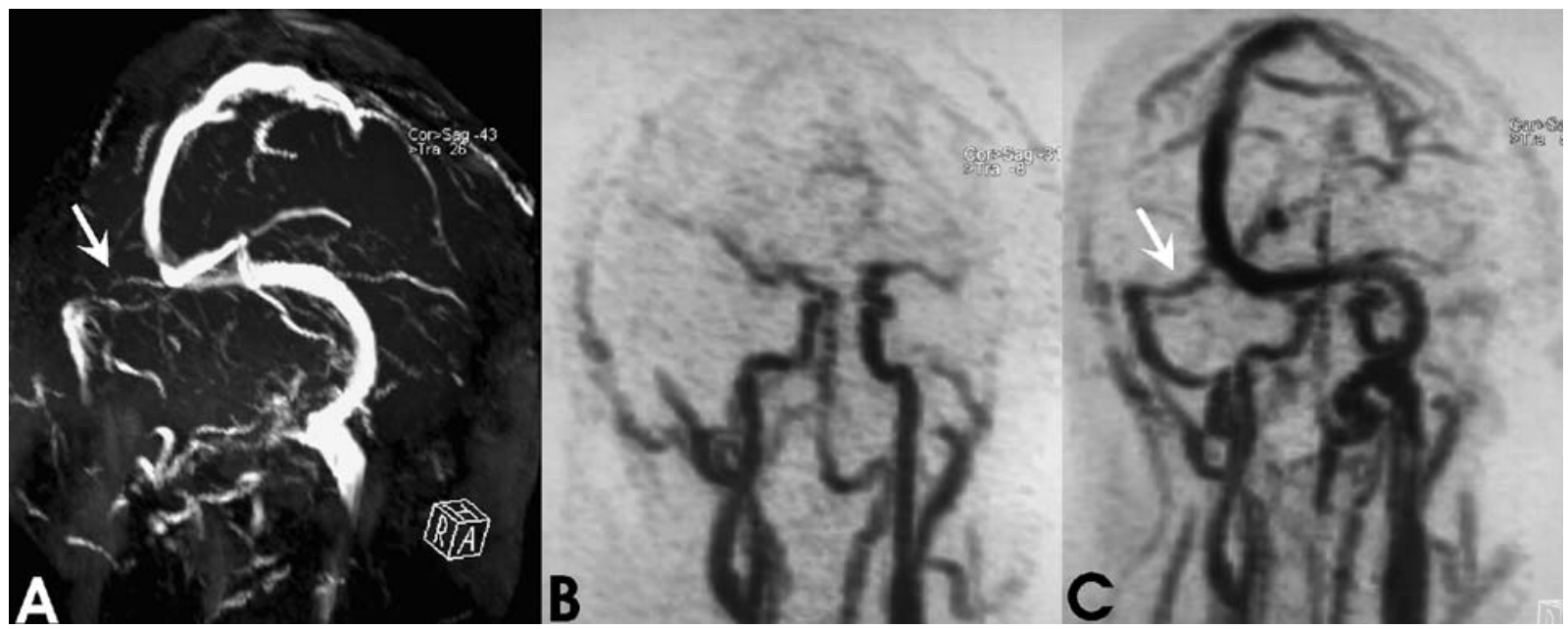

Fig. 3 a Oblique MIP reconstruction of the 2D-TOF venogram reveals a discontinuation of flow signal within the proximal right transverse sinus (white arrow). b,c MIP-reconstructed image of time-resolved 3D-MRA obtained in arterial phase does not show any abnormality with symmetric filling of the cerebral arterial system (b). Flow in the right transverse sinus is definitely shown during venous inflow phase without evidence of sinus obstruction (white arrow, c) 
not possible, time-resolved 3D-MRA revealed retrograde flow to the distal V4 segment. Both time-resolved 3DMRA and DSA showed definitive occlusion of the right VA by the inserted coils.

Patient 8 presented with symptoms of vertebrobasilar insufficiency. Standard MRI and MRA revealed a persistent trigeminal artery. Time-resolved 3D-MRA and subsequent DSA demonstrated the retrograde perfusion of both posterior cerebral arteries and the basilar artery from the anterior circulation. The VAs showed discontinuation in the V4 segment with exclusive bilateral PICA supply. 3D-TOF MRA failed to show these findings, presumably due to saturation effects.

\section{Tumor}

A patient with a hypervascularized tumor (carotid paraganglioma) presented to our institution for endovascular embolization (patient 9, Fig. 5). TOF MRA did not allow an exact assessment of tumor perfusion dynamics due to saturation effects. The time-resolved images demonstrated a strong and early arterial hyperperfusion. Images obtained from the pre-interventional angiogram with selective catheterization of the internal and external carotid arteries demonstrated the arterial supply to the tumor arising exclusively from the ascending pharyngeal artery, which originated as a variant directly from the ICA. Timeresolved 3D-MRA failed to show this information, as did unselective DSA with injection into the common carotid artery.

In patient 10 with a large paraganglioma at the jugular foramen with intra- and extracranial extension that had previously been embolized, time-resolved 3D-MRA demonstrated a strong arterial tumor blush and occlusion of the ipsilateral internal jugular vein. In addition, arterial feeders from ECA branches and venous drainage with retrograde flow in the sigmoid sinus were noted in accordance with findings obtained from DSA. Superselective DSA revealed multiple small feeders from different ECA branches, and small feeders from the VA (V3 segment), ICA (C5 segment) and PICA. 3D-TOF MRA did not differentiate arterial feeders. 2D-TOF MR venography showed signal cancellations within the ipsilateral transverse/sigmoid sinus presumably due to turbulent flow, as neither DSA nor timeresolved MRA revealed any obstruction or occlusion proximal to the tumor.

\section{Discussion}

Noninvasive time-resolved fast 2D-MRA imaging of diseases that present with AV shunting, e.g., DAVFs, AVMs or hypervascularized tumors, has proven to be a promising technique $[4,5,10,11]$. However, inherent limitations of this technique are mainly due to the projective nature of the obtained images. In time-resolved 3D-MRA, required acquisition time is the primary limiting factor for developing a sequence that can provide sufficient spatial and temporal resolution. For example, in a recent report, a dynamic 3D-MRA study for the assessment of DAVFs yielded a temporal resolution of $4 \mathrm{~s}$ per dataset. This time frame allowed the separation of an arterial and a venous phase, but it was considered only marginally adequate for detailed study of cerebral AV dynamics in regard to the short cortical AV transit time of 5-7 s [12].

Exploiting the advantages of parallel imaging techniques, we used a 3D sequence that covered the whole brain and resolved the AV transit time of a contrast bolus. Furthermore, to exploit fully the advantages of 3D imaging, a nearly isovolumetric voxel size was chosen, which allowed free-form interrogation of the dataset. The results from ten patients with different types of disease affecting the craniocervical circulation showed that time-
Fig. 4 a,b Targeted MIP reconstructions (coronal view) of time-resolved 3D-MRA obtained in arterial phase. The right vertebral artery is not filled in the V3 and proximal V4 segments after endovascular occlusion. The left V3/V4 segment is filled during arterial inflow and retrograde perfusion into the tiny distal right V4 segment is demonstrated (b)

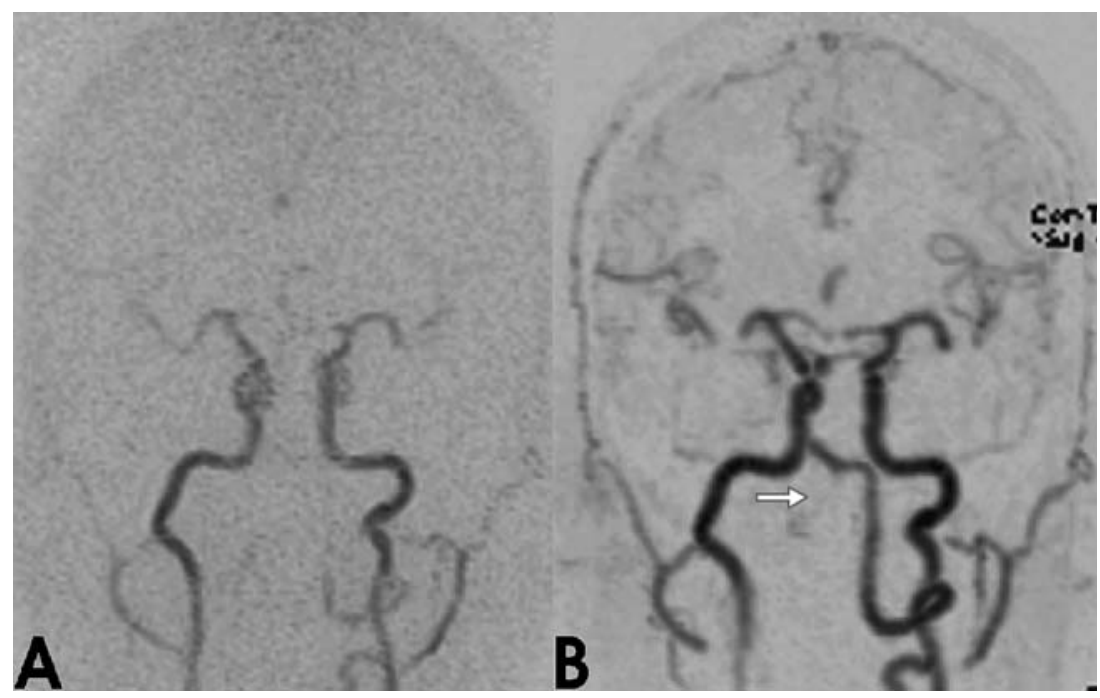




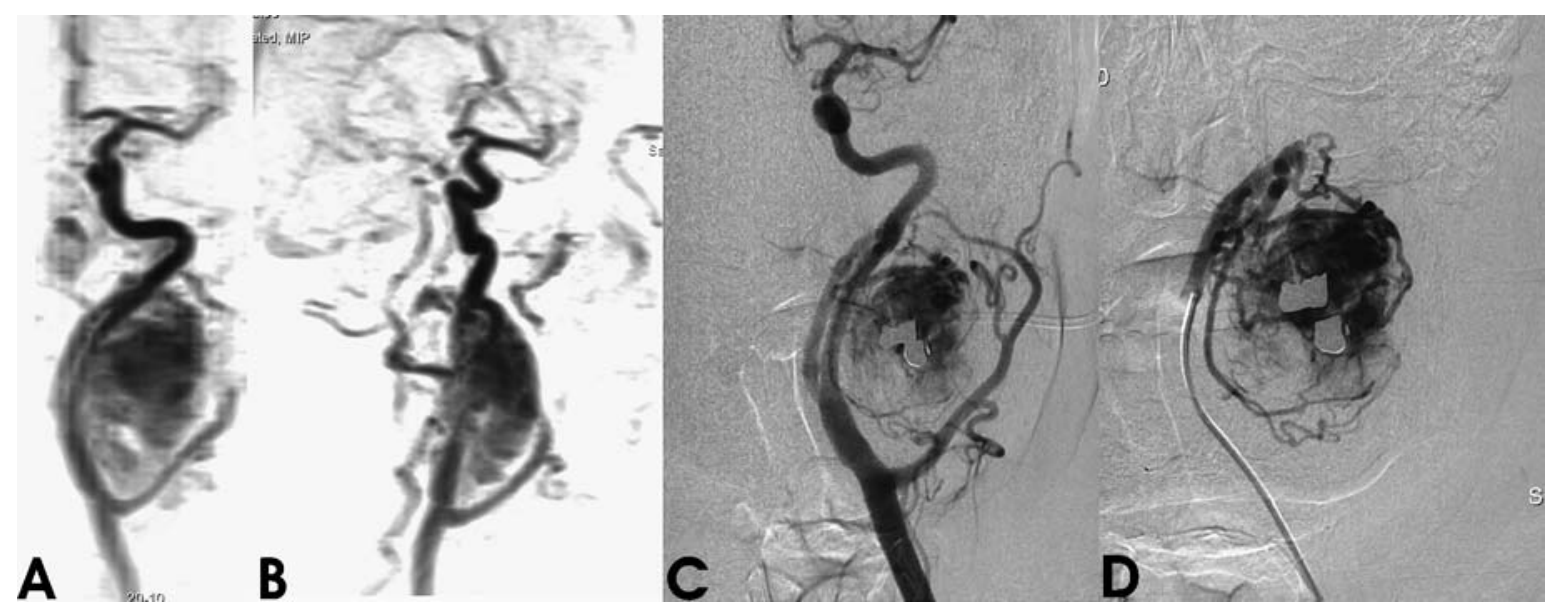

Fig. 5 a,b Time-resolved 3D-MRA images obtained in the early arterial phase (targeted slab acquisition). The tumor filling pattern indicates the strong arterial vascularization. The coronal MIPreconstructed view demonstrates the actual width of the targeted slab (a). Sagittal MIP reconstruction (b) shows tumor extension within

resolved 3D-MRA is valuable as an adjunct to standard MRA techniques because it provides important additional information about the perfusion dynamics of cerebral vessels. An obvious benefit of the time-resolved 3D-MRA technique is the visualization of AV shunts in vascular malformations such as DAVFs. In our cases with AV shunting, time-resolved 3D-MRA provided additional information to standard MR imaging by showing the flow dynamics of these disorders. It was possible, for example, to determine the venous drainage pattern in DAVFs and to demonstrate dominant arterial feeders in these disorders. This is particularly relevant since the grading of venous outflow in a DAVF has been shown to be useful when estimating the individual clinical course of the disease [9].

In patients with steno-occlusive disease, time-resolved 3D-MRA allowed the dynamic visualization of arterial and venous contrast flow. In a case of suspected venous thrombosis, the 2D-TOF venogram showed partial signal loss in the transverse sinus. Although standard MR venography techniques have proven to be valuable in cerebral venous thrombosis, the diagnosis can sometimes be challenging due to various limitations of these methods. In particular, the differentiation of a contrast-enhancing thrombus in chronic dural sinus obliteration from a patent sinus can be demanding in CE MR venography. Loss of signal due to slow flow or in-plane flow in TOF MR venography is a known pitfall of the 2D-TOF technique [8, 13]. In our case, flow in a transverse sinus was clearly demonstrated with time-resolved 3D-MRA. Thus, it may be a promising approach for imaging of venous thrombosis due to its ability to directly visualize the venous passage of a contrast bolus. Time-resolved 3D-MRA allowed depiction of arterial flow dynamics in two cases of occlusion in the posterior circulation, which revealed a suboptimal the carotid bifurcation with spreading of the internal and the external carotid artery. c,d Intraarterial DSA with injection into the common carotid artery confirms strong arterial filling of the tumor (c). Superselective catheterization of an anomalous branch of the internal carotid artery demonstrates the origin of the feeding arteries

visualization on 3D-TOF MRA images due to saturation effects.

Time-resolved 3D-MRA was helpful in analyzing vascularization of tumors as demonstrated in two high-flow paragangliomas. In contrast to TOF angiography which suffered from signal cancellations presumably due to turbulent flow, the arterial and venous dynamics of the tumors were clearly depicted. In both lesions, strong early arterial filling indicated intratumoral high-flow AV shunting.

The target of an isovolumetric information was achieved at the cost of having an in-plane resolution poorer than $1 \times 1$ $\mathrm{mm}$. The lower spatial resolution compared to 3D-TOF MRA and DSA can be limiting for the depiction of small arterial structures, e.g., poor visualization of a tiny arterial lumen (patient 7) and for depicting small peripheral veins. The use of targeted slab scanning may improve the voxel size if the location of the abnormality is known. This technique was applied in three cases, but results were not analyzed and compared in detail as part of this study. In comparison to DSA, time-resolved 3D-MRA does not provide the opportunity to obtain selective or superselective vessel catheterization. Therefore, the differentiation of arterial feeders could not be performed with the same accuracy; for example, a detailed depiction of the arterial supply of hypervascularized tumors was not possible (patients 9 and 10). For a more precise assessment of a single vascular structure or territory, to reduce superimposition of different vascular structures, e.g., arteries and veins, or separate arterial feeders individually placed, targeted MIP reconstructions may be of help. This approach was implemented for better characterization of an AV shunting pattern in a recurrent DAVF (Fig. 2b).

High temporal and spatial resolutions as well as image analysis in different planes are important prerequisites for the precise characterization of complex neurovascular 
diseases. Therefore, technical innovations, e.g., keyhole data sampling or the use of higher magnetic field strength, are of interest for the optimization of dynamic 3D-MRA. Two feasibility studies of the imaging of neurovascular disease with 3D time-resolved MRA implementations on a 1.5-T system using sensitivity encoding (SENSE) and on a 3-T system using GRAPPA have recently been reported $[14,15]$. In contrast to our implementation, non-isovolumetric datasets were generated with comparatively high in-plane resolution but low slice thickness $(0.8 \times 0.8 \mathrm{~mm}$ inplane with a slice thickness of $6 \mathrm{~mm}$ at $3 \mathrm{~T}$; 1.22$1.67 \times 0.49-0.55 \mathrm{~mm}$ in-plane with a slice thickness of $7.5 \mathrm{~mm}$ at $1.5 \mathrm{~T}$ ). Hence, the exploitation of 3D datasets using free-form interrogation for secondary reconstructions, e.g., to obtain individually placed targeted MIP reconstructions, was limited.

In conclusion, isotropic time-resolved 3D-MRA using the GRAPPA reconstruction algorithm provided valuable information about different types of neurovascular disease. It was possible to detect and, to a certain extent, classify vascular malformations. Visualization of AV shunting could be achieved not only at a large dural sinus, but also into cortical veins. From our initial experience with a small group of selected patients, time-resolved 3D-MRA does not have the potential to replace DSA in the initial imaging work-up of vascular malformations or hypervascularized tumors required for therapy planning/clinical management. We were not able to demonstrate benefits for therapy planning in this pilot study. However, apart from screening for DAVFs, time-resolved 3D-MRA might be considered as an alternative for follow-up imaging of endovascularly or surgically treated vascular malformations. In this area of application, it might help in the selection of at-risk patients and prompt further therapy by demonstrating venous hypertension in partially occluded DAVFs.

Using this technique as a non-invasive method for preselection, the number of follow-up DSA examinations after treatment might be reduced, and the use of DSA might be preserved for those patients with DAVFs who will most likely require further treatment. However, to prove these hypotheses, a major study with a larger and more homogeneous number of subjects that includes a statistical evaluation of data is required. Furthermore, in arterial and venous steno-occlusive disease this technique can, to some extent, provide additional information about contrast flow dynamics. Thus, this fast technique may be used as a simple and easy to implement add-on sequence in combination with standard MRA for problem-solving in various types of neurovascular disease.

\section{References}

1. Cloft HJ, Joseph GJ, Dion JE (1999) Risk of cerebral angiography in patients with subarachnoid hemorrhage, cerebral aneurysm, and arteriovenous malformation: a meta-analysis. Stroke 30:317-320

2. Wang Y, Johnston DL, Breen JF et al (1996) Dynamic MR digital subtraction angiography using contrast enhancement, fast data acquisition, and complex subtraction. Magn Reson Med 36:551-556

3. Hennig J, Scheffler K, Laubenberger J, Strecker R (1997) Time-resolved projection angiography after bolus injection of contrast agent. Magn Reson Med 37:145-341

4. Wetzel SG, Bilecen D, Lyrer P et al (2000) Cerebral dural arteriovenous fistulas: detection by dynamic MR projection angiography. AJR Am J Roentgenol 174:1293-1295

5. Aoki S, Yoshikawa T, Hori $\mathrm{M}$ et al (2000) MR digital subtraction angiography for the assessment of cranial arteriovenous malformations and fistulas. AJR Am J Roentgenol 175: 451-453
6. Griswold MA, Jakob PM, Heidemann RM et al (2002) Generalized autocalibrating partially parallel acquisitions (GRAPPA). Magn Reson Med 47 (6):1202-1210

7. Pruessmann KP, Weiger M, Scheidegger MB, Boesiger P (1999) SENSE: sensitivity encoding for fast MRI. Magn Reson Med 42(5):952-962

8. Wetzel SG, Law M, Lee VS, Cha S, Johnson G, Nelson K (2003) Imaging of the intracranial venous system with a contrast-enhanced volumetric interpolated examination. Eur Radiol 13 (5):1010-1018

9. Cognard C, Gobin YP, Pierot L et al (1995) Cerebral dural arteriovenous fistulas: clinical and angiographic correlation with a revised classification of venous drainage. Radiology 194: 671-680

10. Klisch J, Strecker R, Hennig J, Schumacher M (2000) Time-resolved projection MRA: clinical application in intracranial vascular malformations Neuroradiology 42:104-107

11. Coley SC, Romanowski CA, Hodgson TJ, Griffiths PD (2002) Dural arteriovenous fistulae: non-invasive diagnosis with dynamic MR digital subtraction angiography. AJNR Am J Neuroradiol 23:404-407
12. Noguchi K, Melhem ER, Kanazawa T, Kubo M, Kuwayama N, Seto H (2004) Intracranial dural arteriovenous fistulas: evaluation with combined 3D time-offlight MR angiography and MR digital subtraction angiography. AJR Am J Roentgenol 182:183-190

13. Liang L, Korogi Y, Sugahara $T$ et al (2001) Evaluation of the intracranial dural sinuses with a 3D contrastenhanced MP-RAGE sequence: prospective comparison with 2D-TOF MR venography and digital subtraction angiography. AJNR Am J Neuroradiol 22:481-492

14. Ziyeh S, Strecker R, Berlis A, Weber J, Klisch J, Mader I (2005) Dynamic 3D MR angiography of intra- and extracranial vascular malformations at 3T: a technical note. AJNR Am J

Neuroradiol 26:630-634

15. Tsuchiya K, Aoki C, Fujikaw A, Hachiya J (2004) Three-dimensional MR digital subtraction angiography using parallel imaging and keyhole data sampling in cerebrovascular diseases: initial experience. Eur Radiol 14: 1494-1497 